\title{
The risk of cancer in patients with rheumatoid arthritis taking tumor necrosis factor antagonists: a nationwide cohort study
}

\author{
Chun-Ying Wu ${ }^{1,2,3,4}$, Der-Yuan Chen ${ }^{1,4,5,6}$, Jui-Lung Shen ${ }^{7}$, Hsiu J Ho² ${ }^{2}$ Chih-Chiang Chen ${ }^{8}$, Ken N Kuo ${ }^{9,10}$, \\ Han-Nan Liu ${ }^{8}$, Yun-Ting Chang ${ }^{1,8^{*}+}$ and Yi-Ju Chen ${ }^{1,7^{*}+}$
}

\begin{abstract}
Introduction: The association between cancer and use of biologic therapy among rheumatoid arthritis (RA) patients remains controversial. We aimed to compare the relative risk of cancer development between RA patients taking tumor necrosis factor a (TNFa) antagonists and those taking nonbiologic disease-modifying anti-rheumatic drugs (nbDMARDs).

Methods: We conducted a nationwide cohort study between 1997 and 2011 using the Taiwan National Health Insurance Research Database. The risk of newly diagnosed cancer was compared between patients starting TNF-a antagonists (biologics cohort) and matched subjects taking nbDMARDs only (nbDMARDs cohort). Cumulative incidences and hazard ratios (HR) were calculated after adjusting for competing mortality. Standardized incidence ratio (SIR) was calculated for cancer risk. Multivariate analyses were performed using Cox proportional hazards model.

Results: We compared 4426 new users of TNF- $a$ antagonists and 17704 users of nbDMARDs with similar baseline covariate characteristics. The incidence rates of cancer among biologics and nbDMARDs cohorts were 5.35 (95\% confidence interval (Cl) 4.23 to 6.46) and 7.41 (95\% Cl 6.75 to 8.07) per 1000 person-years, respectively. On modified Cox proportional hazards analysis, the risk of cancer was significantly reduced in subjects in biologics cohort (adjusted HR 0.63, 95\% Cl 0.49 to 0.80, $P<.001$ ), after adjusting for age, gender, disease duration, major co-morbidities, and prior use of DMARDs and corticosteroids. However, there was an increased risk for hematologic cancers in biologics cohort, yet without statistical significance. The effect of biologics was consistent across all multivariate stratified analyses and the association between biologics use and cancer risk was independent of dosage of concomitant nbDMARDs.

Conclusion: These findings suggested that RA patients taking TNF-a antagonist are associated with a lower risk of cancer, but not for hematologic cancers, than RA patients taking nbDMARDs alone.
\end{abstract}

\section{Introduction}

Rheumatoid arthritis (RA) affects both patient psychology and physiology, including cardiovascular, musculoskeletal and respiratory systems, and leads to major comorbidities and mortality. RA is associated with certain types of cancer [1-5]. Several studies have indicated

\footnotetext{
*Correspondence: ytchang@vghtpe.gov.tw; yjchenmd@vghtc.gov.tw ${ }^{\dagger}$ Equal contributors

${ }^{1}$ Faculty of Medicine, School of Medicine, National Yang-Ming University, Taipei, Taiwan

${ }^{7}$ Department of Dermatology, Taichung Veterans General Hospital, Taichung, Taiwan

Full list of author information is available at the end of the article
}

an increased incidence of lymphoma [1,3,5-7] and decreased incidence of colorectal and gastric cancer in RA patients [6]. Cancer risk may be related to disease severity and treatment options. For example, the risk of lymphoma is substantially increased in those with high disease activity [8]. On the contrary, non-steroidal anti-inflammatory drugs (NSAIDs) have been reported to be associated with a reduced risk of colon cancer $[9,10]$. Traditional diseasemodifying anti-rheumatic drugs (DMARDs) may also increase the risk of malignancy. For example, discontinuation of methotrexate has been followed by the disappearance of lymphoma in some patients [11].

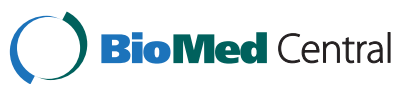

(c) 2014 Wu et al.; licensee BioMed Central Ltd. This is an Open Access article distributed under the terms of the Creative Commons Attribution License (http://creativecommons.org/licenses/by/4.0), which permits unrestricted use, distribution, and reproduction in any medium, provided the original work is properly cited. The Creative Commons Public Domain Dedication waiver (http://creativecommons.org/publicdomain/zero/1.0/) applies to the data made available in this article, unless otherwise stated. 
The introduction of biologic therapies to the management of RA has raised concerns about the risk of cancer, particularly with respect to anti-TNF therapies, due to the role of TNF in tumor progression and surveillance [12]. An experimental animal tumor model has shown that anti-TNF- $\alpha$ antibodies hinder the innate anti-tumor immune responses and promote the growth of immunogenic rat colon tumors that are rejected by immunocompetent untreated rats. It has been suggested that biologics dampen the immune response against normally regressing cancers, thereby fostering malignant growth [13]. The evidence for the association between cancer occurrence and biologic use is conflicting. Systematic review and metaanalyses of clinical trial data have revealed an increased short-term risk of certain cancers in patients taking TNF inhibitors $[14,15]$. On the contrary, a recent meta-analysis of 63 randomized controlled trials demonstrated no association between the use of biologics and cancer risk in RA patients $[16,17]$. To date, only a few observational studies have been conducted on this issue, with controversial results on the long-term cancer risk among users of biologics with RA $[18,19]$.

Here, we aimed to compare the cancer risk among RA patients starting TNF $\alpha$ blocker and those taking nonbiologic disease modifying antirheumatic drug (nbDMARD) in a large cohort of patients with RA, based on a Taiwanese nationwide database.

\section{Methods}

\section{Study design}

We conducted a nationwide cohort study by retrieving all patients with a diagnosis of RA from Taiwan's National Health Insurance Research Database (NHIRD). The NHIRD has been used extensively in epidemiologic studies in Taiwan $[3,20]$. In brief, it consists of detailed health care data from more than 25 million enrollees, representing more than $99 \%$ of Taiwan's entire population. In this database, the diagnostic codes are in the format of the International Classification of Diseases, Revision 9, Clinical Modification (ICD-9-CM). Patients were diagnosed by board-certified physicians in the corresponding specialties. The accuracy of diagnosis of major diseases in the NHIRD, such as stroke and acute coronary syndrome, has been validated. Personal information including body weight, height, family history, laboratory examination results, lifestyle and habits such as smoking and alcohol use was not available from the NHIRD.

This study has been approved by the ethical review board of the Taichung Veterans General Hospital, Taichung, Taiwan. As the datasets used in this study consist of deidentified secondary data released to the public for research purposes, no consent was needed for the review by the ethical review board.

\section{Biologic therapy in the RA cohort}

Biologics were first introduced for RA management in Taiwan in March 2003. Biologics available for RA treatment in Taiwan include TNF- $\alpha$ inhibitors (adalimumab, etanercept and golimumab) and chimeric anti-CD20 monoclonal antibody (rituximab). Other classes of biologics, including humanized IL-6 receptor antibody (tocilizumab, or Actemra ${ }^{\oplus}$ ) and selective T-cell co-stimulatory modulator (abatacept, or Orencia ${ }^{\oplus}$ ), were not covered under the NHI program until mid 2012. Golimumab was not introduced to Taiwan until 1 January 2012. Therefore, none of the patients included in the present study were treated with golimumab, tocilizumab or abatacept.

Under the NHI program, only RA patients with continuous and active disease (fulfilling 1987 American College of Rheumatology (ACR) criteria for RA [21], with disease activity score 28-joint assessment (DAS 28) $>5.1$ points at least twice, recorded at least one month apart, and with radiologic and laboratory evidence) and who failed to respond to at least 6 months treatment with more than two nbDMARDs (mainly methotrexate, cyclosporine, hydroxychloroquine, D-penicillamine, gold salts, et cetera), can apply for reimbursement for biologics. Patients with prior history of pre-malignant or malignant diseases in the past 10 years or those with active infection are not eligible for biologics [22]. Information on medications was retrieved from the pharmacy prescription database. Reliability of the retrieved information was independently verified by two statisticians.

\section{Study cohorts}

All patients with a primary diagnosis of RA (ICD-9CM code 714.0) for the first time and who received nbDMARDs or biologics, including TNF $\alpha$ antagonists and rituximab, between 1997 and 2011 were eligible study subjects. The diagnostic accuracy of RA was confirmed by both specific ICD-9 codes and inclusion in the Registry for Catastrophic Illness Patient Database (RCIPD), a subpart of the NHIRD. As previously described, clinical and laboratory confirmation or typical image presentation of RA is required for patients to be registered in the RCIPD [3].

\section{Identification and definition of study groups}

Patients who received TNF $\alpha$ antagonist, adalimumab or etanercept, or rituximab for RA, were eligible for inclusion in the biologics group. Patients taking nbDMARDs and who had never received prescription for biologics were eligible for inclusion in the nbDMARDs group. We created a matched sample by matching biologics and nbDMARDs subjects by date of birth, age at first use of DMARDs, gender, concomitant comorbidities, duration of disease, and starting date of study, as described below.

Follow up began on the date that a patient added or switched to a TNF- $\alpha$ antagonist (adalimumab or etanercept; 
golimumab was not yet available in 2011 in Taiwan) or rituximab. A matched calendar date as the index date was determined in matched nbDMARD subjects. Only when the index date is determined can we include those having no history of malignancies before or 6 months after the index date from the eligible nbDMARDs cohort. Finally, we selected matched patients in the nbDMARDs cohort fulfilling all of these criteria, including age, gender, comorbidities and those listed above. All participants were observed for the occurrence of outcomes, until death, or 31 December 2011. To study the patients with active disease requiring long-term disease control, we included only those who received nbDMARDs or biologics for at least 3 months after the start of follow up (the index date). Patients receiving follow up for less than 6 months were not included. Patients with history of malignant disease (ICD-9-CM codes: 140-208, ICD-O-3 codes: $\mathrm{C} 00-\mathrm{C} 80)$ before or during the 6 months following the index date were not included.

\section{Main outcome measurements}

Patients with cancer were defined as those having a new diagnosis of cancer (ICD-9-CM codes: 140-208, ICD-O3 codes: $\mathrm{C00}-\mathrm{C} 80$ ) at least 6 months after the index date. To reduce the mixed effect of prior use of nbDMARDs and new biologics, diseases of outcome diagnosed during the first 6 months of observation were not included.

\section{Identification of cancer cases}

We identified the diagnoses of cancers based on the records of the RCIPD. To apply for a cancer catastrophic illness certificate, cytological or pathological reports or evidence including additional laboratory and imaging studies supporting the diagnosis of cancer, such as tumor marker surveys, radiograph, bone scan, computer tomography $(\mathrm{CT})$ scan or magnetic resonance imaging (MRI) scan, must be provided. At least two other oncologists carefully examine the medical records and laboratory information including imaging studies. Only those patients who meet the criteria of diagnoses are issued certificates. We excluded those with in-situ malignancies, as in-situ malignant diseases do not qualify for a catastrophic illness certificate. The diagnostic codes of malignancies were defined as those from 140 to 208.91 in the ICD-9 revision clinical modification format (ICD-O-3 codes: C00-C80). We categorized the cancer cases into hematologic cancers and non-hematologic cancers. Hematologic cancers were subcategorized into leukemias (ICD9-CM codes 204 to 208; ICD-O3 codes: 9811 to $9818,9820,9823,9826,9827$, 9831 to $9837,9840,9860$ to $9861,9863,9865$ to 9867 , 9869, 9870 to $9876,9891,9895$ to $9898,9910,9911,9920$, 9930, 9945, 9946, 9963, 9742, 9800, 9801, 9805 to 9809 , 9931, 9940, 9948, 9964) and lymphomas (including nonHodgkin's lymphoma, multiple myeloma (ICD9-CM codes
200, 202 to 203; ICD-O-3 codes 9590, 9591, 9596, 9597, 9670, $9671,9673,9675,9678$ to $9680,9684,9687$ to 9691 , 9695, 9698, 9699, 9701, 9702, 9705, 9708, 9709, 9712, 9714, 9716 to 9719,9724 to $9729,9735,9737,9738$, 9732 to 9733) and Hodgkin's lymphoma (ICD9-CM code 201; ICD-O-3 codes 9650 to $9655,9659,9663$ to 9665, 9667)), according to the methods of the Cancer Registry in Taiwan.

\section{Potential confounders}

Certain demographic factors, such as age at first use of nbDMARDs, gender, and comorbidities such as hypertension, ischemic heart disease, including myocardial infarction, diabetes, cerebrovascular disease, and chronic liver disease, including liver cirrhosis, were considered potential confounders. These variables were determined over a one-year period before the start of follow up.

Other confounders included use of nbDMARDs, use of corticosteroids, and use of NSAIDs including aspirin, one year prior to the index date, as listed in Table 1 . The use of statins and metformin have been reported to affect the development of certain cancers [23,24], and were also considered covariates.

No information on several potential confounders was available, such as smoking, alcohol use, family history, body mass index, rheumatoid arthritis activity, laboratory status, and educational level.

\section{Statistical analysis}

The demographic data of the study population were first analyzed. Follow up for each subject was measured in numbers of years and began on the date of first prescription of biologics in the biologics group (or the matching calendar date in the nbDMARDs group) and ended on the date of censorship, that is, the date of diagnosis of outcome, death, transfer out or the end of the follow-up period.

As death may result from underlying illness, which may also affect the outcome, it leads to informative censoring in the estimation of the incidence of outcome diseases. Therefore, death occurring prior to outcome was considered a competing risk event. The cumulative incidence of newly diagnosed cancers after adjustment for competing mortality was calculated using a two-step process and tested for equality among the study cohorts. Calculation and comparison of cumulative incidence in the presence of competing risk data ratios was conducted using a modified Kaplan-Meier method and Gray's method [25]. We tested the differences in the full time-to-event distributions between the study groups using the log-rank test. The assumption of proportional hazards was confirmed by plotting the graph of the survival function versus the survival time and the graph of the $\log (-\log$ (survival)) versus the log of survival time. 
Table 1 Demographic characteristics of matched study cohorts

\begin{tabular}{|c|c|c|c|}
\hline Characteristics & $\begin{array}{l}\text { Biologics } \\
(n=4,426)\end{array}$ & $\begin{array}{l}\text { nbDMARDs } \\
(\mathrm{n}=17,704)\end{array}$ & $P$-value \\
\hline Age, $y$, mean (SD) & $53.88(13.08)$ & $53.89(13.09)$ & 0.97 \\
\hline Female gender, $\mathrm{n}(\%)$ & $3813(86.20)$ & $15252(86.20)$ & 1.00 \\
\hline Disease duration before index date, y, median (Q1, Q3) & $9.21(6.84,11.23)$ & $9.20(6.81,11.20)$ & 0.70 \\
\hline Follow-up duration, y, median (Q1, Q3) & $3.30(2.00,5.39)$ & $3.25(1.95,5.29)$ & 0.14 \\
\hline \multicolumn{4}{|l|}{ Outcome } \\
\hline Cancer, n (\%) & $89(2.00)$ & $486(2.70)$ & 0.001 \\
\hline Death before outcome, n (\%) & $192(4.30)$ & $933(5.30)$ & $<0.001$ \\
\hline Overall observation person-years & 16650.63 & 65587.93 & \\
\hline Cancer, incidence rates $(95 \% \mathrm{Cl})$ per 1000 person-years & $5.35(4.23,6.46)$ & $7.41(6.75,8.07)$ & $<0.005$ \\
\hline Number of visits per year during follow ups, median (Q1, Q3) & $13.33(11.61,14.81)$ & $6.04(3.15,9.3)$ & $<0.001$ \\
\hline \multicolumn{4}{|l|}{ Co-morbidities, n (\%) } \\
\hline Hypertension & $1418(32.0)$ & $5672(32.0)$ & 1.00 \\
\hline Chronic liver disease & $813(18.4)$ & $3252(18.4)$ & 1.00 \\
\hline Ischemic heart disease & $496(11.2)$ & $1984(11.2)$ & 1.00 \\
\hline Diabetes & $388(8.8)$ & $1552(8.8)$ & 1.00 \\
\hline Cerebrovascular disease & $96(2.2)$ & $384(2.2)$ & 1.00 \\
\hline \multicolumn{4}{|l|}{ Prior drug use, n (\%) ${ }^{1}$} \\
\hline Methotrexate & $4125(93.2)$ & $8659(48.9)$ & $<0.001$ \\
\hline Sulfasalazine & $3276(74.0)$ & $8531(48.2)$ & $<0.001$ \\
\hline Hydroxychloroquine & $3515(79.4)$ & $10976(62.0)$ & $<0.001$ \\
\hline Glucocorticosteroids & $4003(90.4)$ & $12875(72.7)$ & $<0.001$ \\
\hline \multicolumn{4}{|l|}{ Other systemic drugs use, $\mathrm{n}(\%)^{2}$} \\
\hline Statin & $433(9.8)$ & $2069(11.7)$ & $<0.001$ \\
\hline Metformin & $264(6.0)$ & $1096(6.2)$ & 0.60 \\
\hline NSAID & $4349(98.3)$ & $17041(96.3)$ & $<0.001$ \\
\hline Beta-blockers & $1115(25.2)$ & $4337(24.5)$ & 0.35 \\
\hline \multicolumn{4}{|c|}{ Average dosage of certain DMARDs during follow up, each user, mean $(S D)^{3}$} \\
\hline Methotrexate & $9.1(4.32)$ & $6.25(4.31)$ & $<0.001$ \\
\hline Sulfasalazine & $822.11(652.64)$ & $720.69(575.53)$ & $<0.001$ \\
\hline Hydroxychloroquine & 194.71(137.37) & $187.2(123.95)$ & $<0.001$ \\
\hline Glucocorticosteroids & $4.42(3.6)$ & $3.02(3.21)$ & $<0.001$ \\
\hline
\end{tabular}

${ }^{1}$ Drug users indicate patients using drugs within one year prior to the index date. ${ }^{2}$ Use of drugs at least once per month on average during follow up. ${ }^{3}$ The average dosage is depicted as $\mathrm{mg} /$ day for all these drugs, except methotrexate ( $\mathrm{mg} /$ week). DMARDs, disease modifying anti-rheumatic drugs; N, number; NSAID, non-steroidal anti-inflammatory drugs including aspirin and Cox-2 inhibitors; $Q$, quartile.

We applied a modified Cox proportional hazards model in the presence of a competing risk event to examine the independent association between newly diagnosed cancers and use of biologics [25,26]. Assessment of goodness-of-fit of the models with the stepdown method was carried out to analyze the independent risk factors. The influence of biologics on newly diagnosed cancer was further explored by stratification according to age, gender, disease duration, time from start of follow up, and prior use of DMARDs or corticosteroids.
Sensitivity analyses included (1) focusing on individuals who used adalimumab only; ever used adalimumab; or last use of adalimumab before cancer occurrence; and (2) focusing on individuals who used etanercept only; ever used etanercept; or last use of etanercept before cancer occurrence. These sensitivity analyses were conducted with the purpose of examining whether the main findings were robust to different assumptions.

All data management was performed using SAS 9.2 software (SAS Institute Inc., Cary, NC, USA). Calculations of cumulative incidences and Cox models were carried 
out using the cmprsk package of $\mathrm{R}$ [27]. Calculated results were expressed as the estimated number together with the $95 \% \mathrm{CI}$.

\section{Results}

\section{Demographic characteristics of study cohorts}

We identified 47,531 potentially eligible RA patients from the RCIPD. A total of 2,763 patients who never received DMARDs were excluded. Among the remaining 44,768 subjects, 6,871 patients with a history of biologics use including TNF $\alpha$ antagonists and rituximab were eligible for inclusion in the biologics group and the remaining 37,897 patients who had never used biologics were eligible to be included in the nbDMARDs group. We excluded 2,445 patients in the eligible biologics group who received biologics or traditional DMARDs for less than 3 months; or were followed up for less than 6 months, after starting biologics treatments. Next, we matched four subjects in the eligible nbDMARDs cohort with each subject in the biologics cohort, based on the matching criteria listed in Methods. Finally, the biologics group and the nbDMARDs group consisted of 4,426 and 17,704 patients, respectively, as shown in Figure 1.

The biologics group and nbDMARDs group were similar in demographic characteristics and associated comorbidities (Table 1). In the biologics group, 3,270

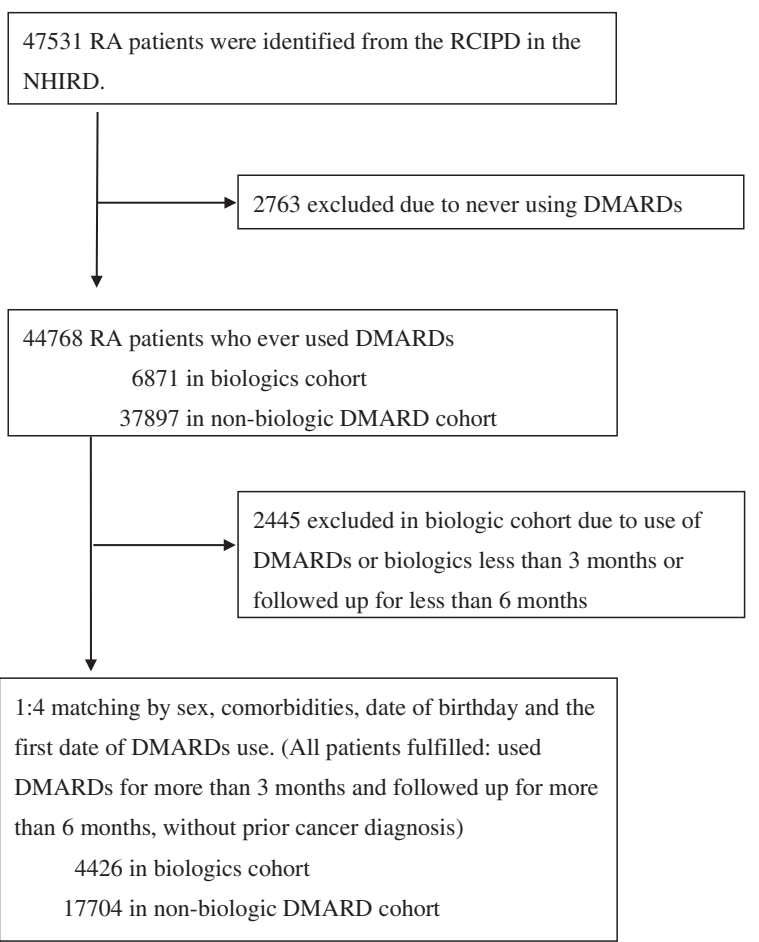

Figure 1 Flow chart of study subject selection. RA, rheumatoid arthritis; RCIPD, Registry for Catastrophic Illness Patient Database; NHIRD, Taiwan National Health Insurance Research Database; DMARD, disease-modifying anti-rheumatic drug. patients (73.9\%) received etanercept, 1,577 patients (35.6\%) received adalimumab and 578 patients (13.1\%) received rituximab. There were 2,529 patients who received etanercept only, 996 patients who received adalimumab only, and 10 patients who received rituximab only. It is not uncommon for biologics to be switched. For example, 323 patients switched from adalimumab to etanercept; 310 patients switched from etanercept to rituximab; 150 patients switched from adalimumab to rituximab; and 108 patients switched treatment among all three biologics.

Disease duration, mean observation time, and number of hospital visits are presented in Table 1. Subjects in the biologics group took more DMARDs and corticosteroids than those in the nbDMARDs group before the index date (Table 1). In addition, more than $92 \%$ of patients in the biologics group received biologics in combination with nbDMARDs or corticosteroids after the index date. The average daily dosages of combined nonbiologic DMARDS in the biologics group were higher than in the nbDMARDs group (Table 1, Additional file 1: Table S1).

\section{Incidence rates of newly diagnosed cancers}

A total of 89 patients in the biologics group and 486 patients in the nbDMARDs group presented with newly diagnosed cancer during the observation period. The 7year cumulative incidence of newly diagnosed cancer after adjusting for competing mortality was significantly lower in the biologics group (3.84\%, 95\% CI 2.91, 4.77) than in the nbDMARDs group $(5.22 \%, 95 \%$ CI 4.69 , 5.75) $(P=0.005)$, as shown in Figure 2.

The incidence rates of newly diagnosed cancer were estimated to be 5.35 per 1000 patient-years (95\% CI 4.23, 6.46) in the biologics group and 7.41 per 1000 patientyears $(95 \%$ CI $6.75,8.07)$ in the nbDMARDs group, with statistically significant difference (Table 2). No cancer cases were observed among those taking rituximab alone. The cancer risk was lower in those taking adalimumab alone, when compared with those taking etanercept alone, but without statistical significance.

\section{Multivariate analysis after adjustment for competing mortality}

When compared with the use of nbDMARDs, starting of TNF- $\alpha$ antagonists was associated with a significantly reduced risk of cancer (adjusted HR 0.63, 95\% CI 0.49, 0.80, $P<0.0001$ ), after adjusting for age, gender, disease duration, number of hospital visits, prior use of DMARDs, prior use of systemic corticosteroids, and presence of diabetes, hypertension, ischemic heart disease, cerebrovascular disease, or chronic liver disease (Table 3).

Use of statins, use of metformin, use of beta-blockers, long disease-duration and presence of ischemic heart 

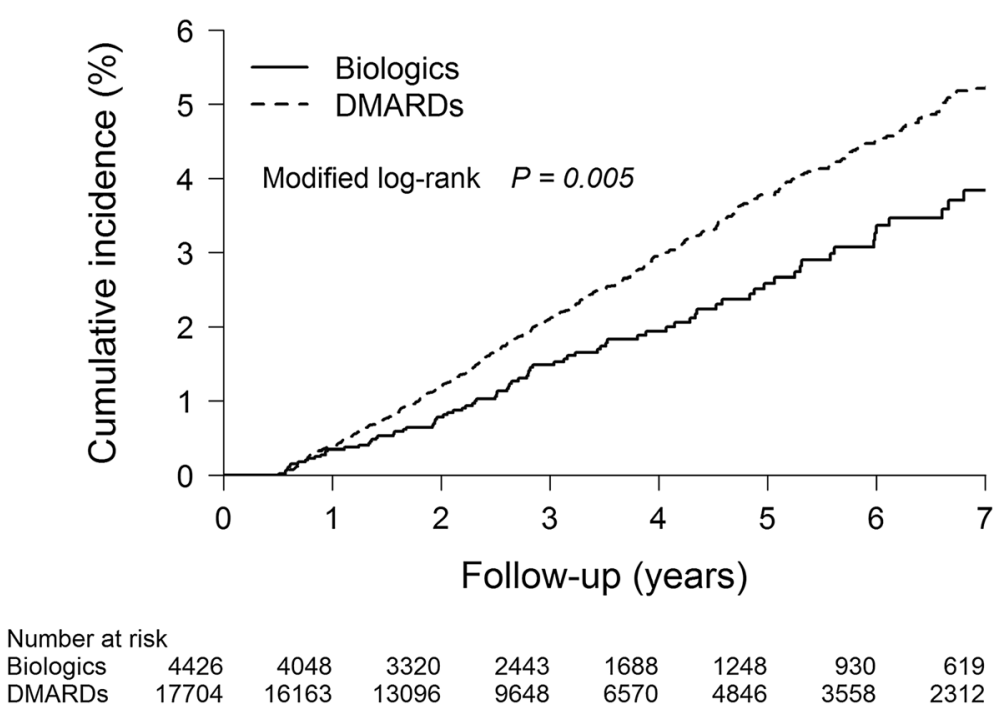

Figure 2 Cumulative incidences of new cancer occurrence following initiation of biologics after adjustment for competing mortality. Calculation and comparison of cumulative incidences in the presence of competing risk data ratios were conducted using a modified Kaplan-Meier method and Gray's method. New cancer occurrence during the first six months was excluded. DMARDs, disease-modifying anti-rheumatic drugs.

disease were also associated with a lower risk of cancer. On the contrary, increasing age and higher average number of hospital visits were significantly associated with cancer occurrence (Table 3).

\section{Multivariate stratified analyses after adjustment for competing mortality}

We conducted multivariate stratified analyses adjusted for competing mortality to determine the effects of biologics on cancer development among different subsets of RA patients. A negative association between biologics use and cancer occurrence was observed in almost all subsets of study subjects, especially among those of older age, of female gender, with long-disease duration, and free of comorbidities (Figure 3).

To examine the potential mixed effect of DMARDs or corticosteroids on cancer risk in biologics users, we further conducted analyses by stratifying the dose of concomitant DMARDs. The association between biologics use and cancer risk was independent of daily dosage of concomitant nbDMARDs.

\section{Specific cancer risks and sensitivity analyses by} standardized incidence ratio (SIR)

Sensitivity analyses focusing on patients receiving different treatment patterns of TNF- $\alpha$ inhibitors is presented

Table 2 Multivariate analyses of risk of malignant diseases among biologic users in comparison with matched controls

\begin{tabular}{|c|c|c|c|c|c|c|}
\hline & & All & TNF-a inhibitor ${ }^{1}$ users & Adalimumab $^{2}$ & Etanercept $^{3}$ & Rituximab \\
\hline \multirow[t]{3}{*}{ Biologics } & Event, number & 89 & 89 & 8 & 71 & 0 \\
\hline & Total person-years & 16650.63 & 16624.36 & 2203.45 & 10363.75 & 26.27 \\
\hline & IR per $1000(95 \%$ Cl) & $5.35(4.23,6.46)$ & $5.35(4.24,6.47)$ & $3.63(1.11,6.15)$ & $6.85(5.26,8.44)$ & - \\
\hline \multirow[t]{6}{*}{ nbDMARDs ${ }^{4}$} & Event, number & 486 & 485 & 51 & 309 & 1 \\
\hline & Total person-years & 65587.93 & 65485.91 & 8763.78 & 41209.78 & 102.02 \\
\hline & IR per $1000(95 \%$ Cl) & $7.41(6.75,8.07)$ & $7.41(6.75,8.07)$ & $5.82(4.22,7.42)$ & $7.50(6.66,8.33)$ & $9.80(0.00,29.01)$ \\
\hline & $\mathrm{HR}(95 \% \mathrm{Cl})$ & $0.63(0.49,0.80)$ & $0.63(0.50,0.81)$ & $0.61(0.28,1.33)$ & $0.83(0.62,1.10)$ & - \\
\hline & $\operatorname{IRR}(95 \% \mathrm{Cl})$ & $0.72(0.58,0.90)$ & $0.72(0.58,0.91)$ & $0.62(0.30,1.31)$ & $0.91(0.71,1.18)$ & - \\
\hline & $P$-value & 0.0046 & 0.0049 & 0.2147 & 0.4926 & - \\
\hline
\end{tabular}

${ }^{1}$ Includes all patients who have used TNF-a inhibitors, with or without disease-modifying anti-rheumatic drugs (DMARDs) or corticosteroids, in comparison with matched controls. ${ }^{2}$ Use of adalimumab alone, with or without DMARDs or corticosteroids, in comparison with matched controls. ${ }^{3}$ Use of etanercept alone, with or without DMARDs or corticosteroids, in comparison with matched controls. ${ }^{4}$ Indicates the event number and total observed person-years of matched subjects from THE nonbiologic DMARDS (nbDMARDs) cohort for each of those using different types of TNF- $\alpha$ inhibitors and rituximab. HR, hazard ratio; IR, incidence rate; IRR, incidence rate ratio. 
Table 3 Death-adjusted multivariate analyses of cancer risk in both study cohorts

\begin{tabular}{|c|c|c|c|}
\hline Covariate & $\begin{array}{l}\text { Adjusted } \\
\text { hazard ratio }^{4}\end{array}$ & $95 \% \mathrm{Cl}$ & $P$-value \\
\hline Biologics use & 0.63 & $0.49,0.80$ & $<0.001$ \\
\hline Male gender & 1.10 & $0.88,1.38$ & 0.39 \\
\hline Age at start, per year & 1.04 & $1.03,1.05$ & 0.000 \\
\hline Disease duration ${ }^{1}$, per year & 0.96 & $0.94,0.99$ & 0.02 \\
\hline $\begin{array}{l}\text { Number of hospital visits, } \\
\text { per year during observation }\end{array}$ & 1.01 & $1.01,1.02$ & 0.000 \\
\hline Methotrexate $^{2}$ & 1.04 & $0.86,1.26$ & 0.68 \\
\hline Salfulsalazine ${ }^{2}$ & 1.01 & $0.84,1.20$ & 0.93 \\
\hline Hydroxychloroquine $^{2}$ & 1.13 & $0.94,1.36$ & 0.19 \\
\hline Corticosteroids $^{2}$ & 1.03 & $0.84,1.28$ & 0.75 \\
\hline Diabetes mellitus & 1.26 & $0.92,1.74$ & 0.15 \\
\hline Ischemic heart disease & 0.70 & $0.54,0.92$ & 0.01 \\
\hline Cerebrovascular disease & 0.75 & $0.45,1.26$ & 0.28 \\
\hline Hypertension & 1.21 & $0.98,1.50$ & 0.07 \\
\hline Chronic liver disease & 1.16 & $0.94,1.42$ & 0.17 \\
\hline Statins $^{3}$ & 0.64 & $0.48,0.86$ & 0.003 \\
\hline Metformin $^{3}$ & 0.67 & $0.45,1.00$ & 0.05 \\
\hline NSAIDs $^{3}$ & 0.94 & $0.54,1.64$ & 0.83 \\
\hline Beta-blocker $^{3}$ & 0.77 & $0.63,0.93$ & 0.008 \\
\hline
\end{tabular}

${ }^{1}$ Disease duration represented by duration from the first disease-modifying anti-rheumatic drugs (DMARDs) prescription for rheumatoid arthritis to the index date. ${ }^{2}$ Use of drugs within one year prior to the index date. ${ }^{3}$ Use of drugs at least once per month on average during follow-up. ${ }^{4}$ Modified hazard ratio adjusted by multiple covariates including age, gender, disease duration, prior history of hypertension, diabetes, ischemic heart disease, cerebrovascular disease, chronic liver diseases, use of DMARDs within one year prior to the index date, corticosteroids, aspirin, non steroidal anti-inflammatory drugs (NSAIDs), statins and metformin.

in Additional file 2: Table S2. We performed SIR estimation to compare the cancer risks of both study groups with the general population. The cancer risk was significantly elevated among subjects in the nbDMARDs cohort when compared with the general population, both for non-hematologic cancers (SIR 1.31, 95\% CI 1.19, 1.44) and hematologic cancers (SIR 2.28, 95\% CI 1.55, 3.24) (Table 4). On the contrary, the overall cancer risk for subjects in the biologics cohort was comparable to that of the general population (SIR 0.97, 95\% CI 0.78, 1.19). The overall cancer risk in those taking adalimumab was slightly lower than in those taking etanercept, either in the patterns of treatment alone, ever-treated or last-treated with the biologics of interest, yet without statistical significance (Table 4, Additional file 2: Table S2).

However, the risk of hematologic cancer was significantly elevated in the biologics cohort when compared with the general population (SIR 4.64, 95\% CI 2.65, 7.53) (Table 4). The risk of non-Hodgkin's lymphoma was comparable between those taking adalimuamb and those taking etanercept, either in the patterns of treated alone, ever-treated or last-treated with the biologics of interest, without statistical significance (Additional file 2: Table S2).

\section{Discussion}

Our study provided nationwide-based evidence that starting TNF- $\alpha$ antagonists does not increase, but rather decreases, the risk of cancer for RA patients, in comparison with those taking nbDMARDs alone, with a reduction in 7-year cumulative incidence from $5.22 \%$ to $3.84 \%$. The effect of TNF- $\alpha$ antagonists was statistically significant among almost all subgroups of patients. The association between TNF- $\alpha$ antagonists and cancer risk was independent of daily dosage of concomitant nbDMARDs.

Controversy remains with respect to whether biologics carry a cancer risk for RA patients. A meta-analysis that included nine RCTs and 5,005 subjects conducted by Bongartz et al. [15] demonstrated a dose-dependent increased risk of malignancy in RA patients treated with TNF inhibitors, mainly infliximab and adalimumab. On the contrary, the most recent systemic review and metaanalysis from 63 RCTs that included 29,423 patients revealed no statistically significant increased risk of any type of cancer with use of TNF antagonists plus nbDMARDs, in comparison with use of nbDMARDs alone [16]. The results from the British Society for Rheumatology Biologics Registry for RA patients demonstrated no difference in risk of solid cancer among patients receiving TNF- $\alpha$ antagonists and patients receiving nbDMARDs over a 5-year observation period. That study also demonstrated no difference in the relative risk of cancer for any of the individual TNF- $\alpha$ antagonists [28]. Two small clinical trials in Taiwan reported a negative association with short-term (12-week) risk for cancer in RA patients using etanercept and adalimumab, compared to RA patients taking methotrexate $[29,30]$. Several other clinical trials have reported controversial results for long-term cancer risk in subjects taking TNF- $\alpha$ antagonists, with an observation time of up to 104 weeks [31-35]. However, half of the trials only included subjects with active early RA (less than 2 years).

The association between lymphoma occurrence and use of biologics in RA has been of major concern [7,17,36,37]. In the present study, we demonstrated a significantly elevated risk of lymphoma in both the biologics cohort and nbDMARDs cohort when compared with the general population. Higher risk of lymphoma was observed in the biologics cohort when compared with the nbDMARDs cohort, with statistical significance (Table 4, Additional file 2: Table S2). Data from the MedWatch postmarketing adverse event surveillance system of the Food and Drug Administration have revealed an association between lymphoma and treatment with etanercept or infliximab 


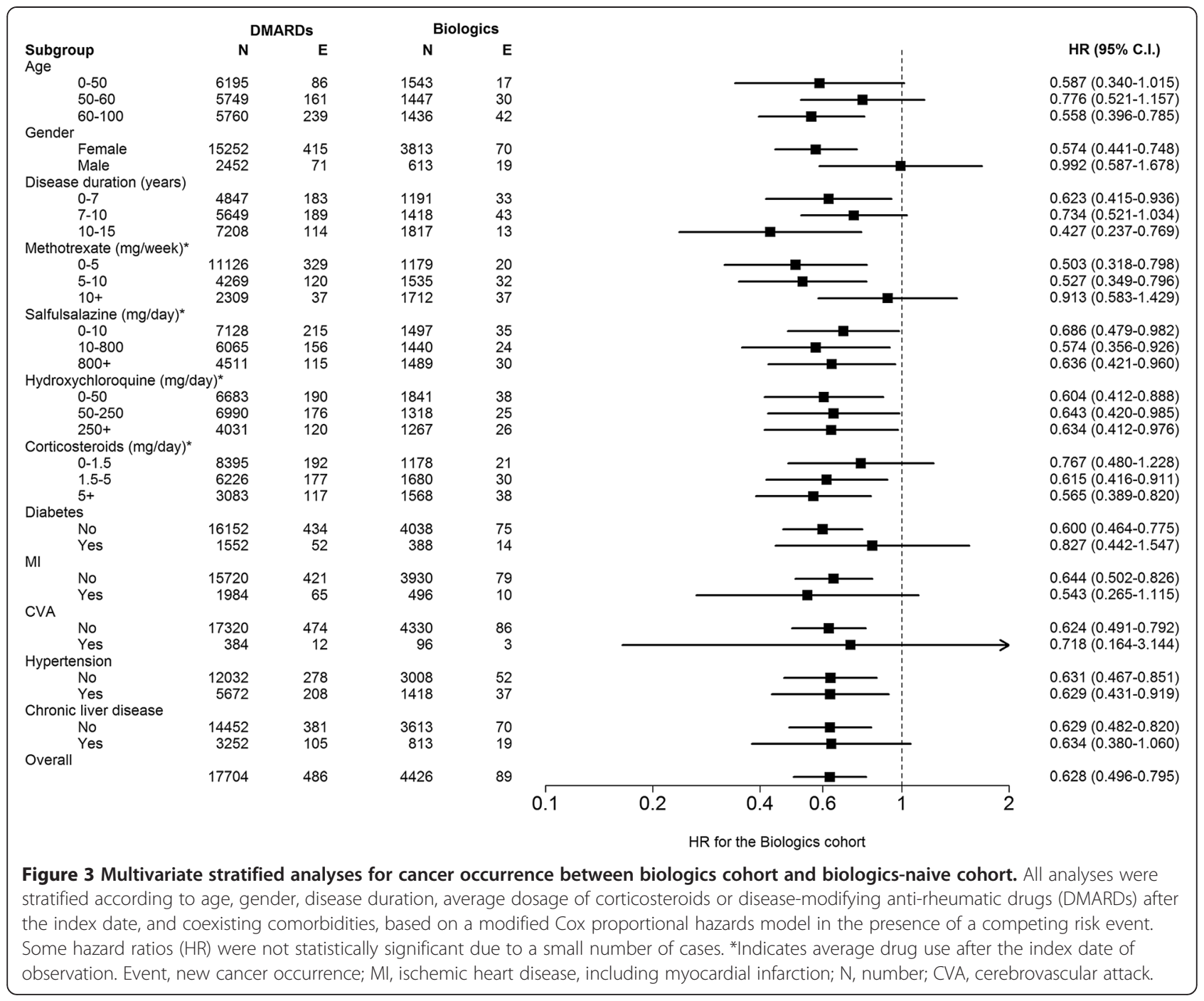

[38]. Recent meta-analyses of 71 global clinical trials investigating the safety profiles of adalimumab across several entities of arthritis have indicated a slightly increased risk of lymphoma among adalimumab users with RA when compared with the general population [39]. However, a study comparing published case reports and reports from the French pharmacovigilance system has argued the link between lymphoma and TNF- $\alpha$ antagonists [40]. A prospective cohort study with a large sample size and a long duration of follow up is required to examine the causality between biologics and cancer development.

The association between malignant disease and chronic inflammation is well-documented [41]. We assumed that patients taking biologics had more severe disease and were more likely to develop malignant diseases. On the contrary, the chance of developing cancer was reduced in patients taking biologics, in comparison with matched patients taking nbDMARDs only. This implies that the use of biologics attenuates the disease activity and then reduces the risk of cancer development to a level that is even lower than in subjects taking nbMDARDs only.

The mixed effect of concomitant DMARDs may also affect the risk of cancer. Physicians in Taiwan usually reserve low-dose DMARDs in combination with biologics for reducing the chance of auto-antibody production and improving clinical outcomes. Our subjects in the biologics cohort received a higher daily dosage of DMARDs than those in the matched nbDMARDs cohort (Table 1). These results suggested that patients in the biologics cohort have more severe or refractory disease than those in the nbDMARDs cohort. Further multivariate stratified analyses suggested that the association between biologics use and cancer risk is independent of dosage of concomitant non-biologic DMARDs. This further emphasizes the additional beneficial role of biologics in patients with severe $R A$.

A low prevalence of RA has been reported in Asian countries [42-44]. Based on the estimate from the Taiwan 
Table 4 Standardized incidence ratio (SIR) of specific cancer types among subjects in the biologics cohort and nonbiologic DMARDs cohort

\begin{tabular}{|c|c|c|c|c|c|c|}
\hline \multirow[t]{2}{*}{ Cancer origin } & \multicolumn{2}{|c|}{ Biologics } & \multirow[t]{2}{*}{ SIR $(95 \% \mathrm{Cl})$} & \multicolumn{2}{|c|}{ nbDMARDs } & \multirow[t]{2}{*}{$\operatorname{SIR}(95 \% \mathrm{CI})$} \\
\hline & $\mathrm{N}$ & Expected & & $\mathrm{N}$ & Expected & \\
\hline Non-hematologic cancers & 73 & 88.46 & $0.83(0.65,1.04)$ & 455 & 347.47 & $1.31(1.19,1.44)$ \\
\hline Oral cavity & 2 & 4.55 & $0.44(0.05,1.59)$ & 20 & 17.92 & $1.12(0.68,1.72)$ \\
\hline Digestive system ${ }^{1}$ & 20 & 32.03 & $0.62(0.38,0.97)$ & 135 & 125.75 & $1.07(0.90,1.27)$ \\
\hline Lungs $^{2}$ & 11 & 10.39 & $1.06(0.53,1.89)$ & 57 & 40.77 & $1.40(1.06,1.81)$ \\
\hline Breast, cervix ${ }^{3}$ & 22 & 19.96 & $1.10(0.69,1.67)$ & 115 & 78.45 & $1.47(1.21,1.76)$ \\
\hline Bladder/kidney ${ }^{4}$ & 14 & 15.75 & $0.89(0.49,1.49)$ & 96 & 61.81 & $1.55(1.26,1.90)$ \\
\hline Melanoma & 0 & 0.21 & - & 0 & 0.83 & - \\
\hline NMSC & 5 & 2.43 & $2.05(0.66,4.79)$ & 15 & 9.58 & $1.57(0.88,2.58)$ \\
\hline Unspecified origin & 4 & 5.79 & $0.69(0.19,1.77)$ & 32 & 22.76 & $1.41(0.96,1.99)$ \\
\hline Hematologic cancers ${ }^{5}$ & 16 & 3.45 & $4.64(2.65,7.53)$ & 31 & 13.57 & $2.28(1.55,3.24)$ \\
\hline Lymphoma & 13 & 2.12 & $6.13(3.26,10.49)$ & 21 & 8.34 & $2.52(1.56,3.85)$ \\
\hline $\mathrm{NHL}$ & 12 & 2.05 & $5.86(3.02,10.24)$ & 20 & 8.06 & $2.48(1.52,3.83)$ \\
\hline $\mathrm{HL}$ & 1 & 0.07 & $13.97(0.18,77.7)$ & 1 & 0.28 & $3.56(0.05,19.79)$ \\
\hline Leukemia & 1 & 1.33 & $0.75(0.01,4.18)$ & 9 & 5.23 & $1.72(0.79,3.27)$ \\
\hline
\end{tabular}

${ }^{1}$ Cancers of digestive system include cancers of the stomach, colon, liver, pancreas. ${ }^{2}$ Including cancers of the lungs, bronchus and pleura. ${ }^{3}$ Including cancers of the uterus and other female reproductive organs. ${ }^{4}$ Including cancers of the prostate, testis and other male reproductive organs. ${ }^{5}$ Myeloma not included. $\mathrm{NbDARDS}$, nonbiologic disease-modifying anti-rheumatic drugs; HL, Hodgkin's lymphoma; NHL, non-Hodgkin's lymphoma; NMSC, non-melanoma skin cancers; $\mathrm{N}$, number.

Rheumatology Association and nationwide epidemiologic studies, the prevalence of RA is between 0.2 and $0.4 \%$ of the Taiwanese general population [42]. Likewise, the prevalence of RA is less than $0.3 \%$ in China [43] and decreased from $0.54 \%$ in 1969 to $0.17 \%$ in 1996 in Japan [44]. These prevalence estimates are all much lower than the estimated $1 \%$ prevalence in Western countries. The estimate of prevalence in the present study (approximately 0.2\%) is consistent with that of prior studies in Taiwan. However, as we included only patients fulfilling all diagnostic criteria for RA, some patients with very early RA, not registered in the RCIPD, may not have been included.

The incidence rates of cancer in RA have been reported to be 4.58 in Korea and 6.7 in Taiwan per 1000 patient-years, which are lower than in Western populations $[45,46]$. The incidence rates of all-site malignancies in TNF- $\alpha$ antagonist users in Western populations have been reported to range from 4.7 to 13 per 1000 patientyears $[18,19,47]$. More recently, Mercer et al. reported the incidence rate of cancer in TNF- $\alpha$ antagonist users to be 8.1 per 1000 patient-years, compared with 11.7 per 1000 patient-years in nbDMARDs users [28]. The results from the present study are consistent with those of prior studies. More epidemiologic studies are required to determine the cancer prevalence in more Asian populations.

On multivariate analysis, more hospital visits were positively associated with cancer occurrence. Patients with more hospital visits were supposed to have more chance of cancer surveillance. Although there were more hospital visits among subjects in the biologics cohort than the matched nbDMARDs cohort, as presented in Table 1, our results further reinforced the link between biologics use and a lower risk of cancer.

Patients with ischemic heart disease (IHD) were found to have a negative association with cancer occurrence on multivariate analyses. Patients with IHD tended to have fatal cardiovascular outcomes and might die too early to develop a new cancer. After matching comorbidities in both study cohorts, this bias may happen randomly in both cohorts. In addition, the medications commonly used for IHD may play a role in preventing certain cancer occurrence or recurrence [48-50]. The negative association is observed independently for use of statins, and NSAIDs, including aspirin or beta-blockers, on multivariate analyses. This link is worth further investigation. Different study designs would be needed to determine the role of other anti-hypertensive drugs on cancer risk.

The strengths of the current study include the utilization of the nationwide NHIRD, which contains detailed pharmacy claims for each study subject and is widely accepted for epidemiological studies $[3,20,46]$. To eliminate immortal time bias, we applied incident user design to this study, with the exposure time calculated from the start of new biologics in the biologics group (and corresponding date in the nbDMARDs group). In addition, we matched both biologics and comparator groups for age, gender, propensity score, date of first use of DMARDs, and date of first use of biologics. An active comparator cohort, as was used in this 
study, serves to restrict analyses to patients with active long-term disease of similar severity and with similar comorbidity index.

There are several limitations to the present study. It is difficult to infer causation between a drug of interest and risk of cancer occurrence based on an observational study, without a random assignment of treatments. Confounding by indication may exist and account for differences in outcomes. In addition, the patients in the study cohorts may differ in many measured and unmeasured ways. We did not have personal information such as lifestyle, family history of malignant diseases, body mass index, laboratory or serologic information, and records of disease severity score, all of which may contribute to cancer risk. To avoid these biases, we selected only patients with matched age, gender, disease duration, and concomitant comorbidities. Multivariable analysis was performed to adjust for potential confounders. Furthermore, we conducted multivariable stratified analysis to examine the risk of cancer for the study cohorts in different strata. Although unmeasured confounders may still exist, we believe the methodology used in the present study is solid and robust. To meet the eligibility for biologics use, we selected only subjects free of cancer before taking biologics or the start of follow up, which may have led to an underestimation of cancer incidence in both groups.

Coding error is possible in a database. To minimize this bias, we enrolled only patients from the catastrophic illness database who met the criteria for RA and malignant diseases. Furthermore, most patients undergo regular physical and laboratory examination during observation. A surveillance bias may contribute to an increased frequency of cancer. Our analyses were adjusted for number of hospital visits to prevent this bias. Some patients may have used self-paid biologics and thus may have been inappropriately classified into the nbDMARDs cohort. This potential misclassification may have led to an underestimation of the association.

Nonetheless, our study provides important evidence of the safety, in terms of cancer risk, of anti-TNF- $\alpha$ antagonists in RA patients who survive long-term disease and who undergo long-term DMARD treatment. In addition, the potential beneficial effect of biologics was most significant among those of female gender, those with long disease-duration and those free of major comorbidities. The association between biologics and cancer risk was independent of daily dosage of concomitant nbDMARDs.

The results of our study should be interpreted cautiously because they refer only to two out of five available TNF- $\alpha$ antagonists. The association between cancer risk and other TNF- $\alpha$ antagonists needs further analysis. Moreover, these results refer only to RA patients without prior malignant diseases. The investigation of the effect of biologics on patients with existing malignancy requires a different study design.

\section{Conclusion}

The current study suggested that the addition of antiTNF- $\alpha$ therapy is safe, in terms of cancer risk, for RA patients undergoing long-term DMARD treatment, based on a Taiwanese nationwide population.

\section{Additional files}

Additional file 1: Table S1. Comparison of non-biologics diseasemodifying anti-rheumatic drug use prior to the index date between the two study groups.

Additional file 2: Table S2. Sensitivity analyses for the cancer risk among different biologics treatment settings.

\section{Abbreviations}

ACR: American College of Rheumatology; DAS28: Disease activity score 28-joint assessment; DMARD: disease-modifying anti-rheumatic drug; HR: hazard ratio; ICD-9-CM: International Classification of Diseases, Revision 9, Clinical Modification; nbDMARD: nonbiologic disease-modifying anti-rheumatic drug; NHIRD: Taiwan national Health Insurance Research Database; NSAID: non-steroidal anti-inflammatory drug; RA: rheumatoid arthritis; RCIPD: Registry for Catastrophic Illness Patient Database;

TNFa: tumor necrosis factor a.

\section{Competing interest}

The authors declare that they have no competing interest.

\section{Authors' contributions}

CYW, YTC, and YJC conceived the study, provided the concepts, designed the study protocol, acquired the data, analyzed and interpreted the data, and drafted and revised the paper. DYC participated in the design of the study protocol, interpretation of the data, revision of the important intellectual content and drafting of the manuscript; CCC, KNK, HNL and JLS contributed to the data interpretation, revision of the important intellectual content, and drafting of the manuscript. HJH performed the statistical analyses, analyzed the data, and provided intellectual content. All authors have read and approved the final version of the manuscript.

\section{Authors' information}

Professor CY Wu currently serves as the Vice Secretary General of The Gastroenterological Society of Taiwan (2012 to present), as well as on the Board of Directors (2012 to present), Taiwan Liver Cancer Association and Board of Directors (2007 to present), Taiwan Evidence-based Medicine Association. His main research interests include chemoprevention for malignancies including gastric cancer, hepatocellular carcinoma, and autoimmune diseases such as psoriasis and rheumatoid arthritis. His recent work has been published in several top journals in different fields including JAMA, J Clin Oncol, Gastroenterology, Hepatology, Gut, Arthritis Res Ther, Arthritis Rheum, et cetera. Professor DY Chen, the Director of Department of Medical Research, Taichung Veterans General Hospital, currently serves as the President of Taiwan Rheumatology Association (from 2014) and has served on the Board of Directors, The Chinese Society of Immunology. He has conducted many clinical and basic studies of adult-onset Still's disease, rheumatoid arthritis and systemic lupus erythematosus, and his work has been published in many leading journals including Ann Rhem Dis. He is a leader in research and education in the fields of immunology and rheumatology in Taiwan. Professor YT Chang, the Director of Department of Dermatology, National Yang-Ming University, currently serves on the Board of Directors (2012 to present), Taiwanese Dermatological Association. His research interests include clinical and basic research about the association of multiple comorbidities in psoriasis, cutaneous amyloidosis and other autoimmune diseases associated comorbidities, mainly cardiovascular diseases and metabolic syndrome and cancer. His recent work has been published in leading journals in the fields of Dermatology, Internal Medicine 
and Rheumatology. Professor YJ Chen is a senior attending physician in the department of Dermatology of Taichung Veterans General Hospital, and currently serves on the Board of Directors (2009 to the present), Taiwanese Dermatological Association. Her research interests include clinical and basic research about psoriasis and associated comorbidities, mainly cardiovascular diseases and metabolic syndrome. Her recent work has been published in top journals in the fields of Dermatology, Internal Medicine and Rheumatology.

\section{Acknowledgements}

This study has been supported by grants from Taichung Veterans General Hospital (TCVGH-1016802C, -1016801B) and Taipei Veterans General Hospital (R-1100401 and R-11004-02). This study is based on data from the National Health Insurance Research Database provided by the Bureau of National Health Insurance, Department of Health and managed by the National Health Research Institute. The interpretations and conclusions contained herein do not represent those of the Bureau of National Health Insurance, Department of Health or the National Health Research Institute. Yi-Ju Chen has full access to all of the data in the study and takes responsibility for the integrity of the data and the accuracy of the data analysis.

\section{Author details}

'Faculty of Medicine, School of Medicine, National Yang-Ming University, Taipei, Taiwan. ${ }^{2}$ Division of Gastroenterology, Taichung Veterans General Hospital, Taichung, Taiwan. ${ }^{3}$ Department of Public Health and Graduate Institute of Clinical Medicine, China Medical University, Taichung, Taiwan. ${ }^{4}$ Department of Life Sciences, National Chung-Hsing University, Taichung, Taiwan. ${ }^{5}$ Department of Allergy, Immunology and Rheumatology, Taichung Veterans General Hospital, Taichung, Taiwan. ${ }^{6}$ Institute of Microbiology and Immunology, Chung-Shan Medical University, Taiwan, Taiwan. ${ }^{7}$ Department of Dermatology, Taichung Veterans General Hospital, Taichung, Taiwan. ${ }^{8}$ Department of Dermatology, Taipei Veterans General Hospital, Taipei, Taiwan. ${ }^{9}$ College of Medicine, Taipei Medical College, Taipei, Taiwan. ${ }^{10}$ Institute of Population Health Sciences, National Health Research Institutes, Miaoli, Taiwan.

Received: 14 February 2014 Accepted: 2 September 2014 Published online: 30 September 2014

\section{References}

1. Askling J, Fored CM, Baecklund E, Brandt L, Backlin C, Ekbom A, Sundström C, Bertilsson L, Cöster L, Geborek P, Jacobsson LT, Lindblad S, Lysholm J, Rantapää-Dahlqvist S, Saxne T, Klareskog L, Feltelius N: Haematopoietic malignancies in rheumatoid arthritis: lymphoma risk and characteristics after exposure to tumour necrosis factor antagonists. Ann Rheum Dis 2005, 64:1414-1420.

2. Askling J, Fored CM, Brandt L, Baecklund E, Bertilsson L, Feltelius N, Cöster L, Geborek P, Jacobsson LT, Lindblad S, Lysholm J, Rantapää-Dahlqvist S, Saxne T, Klareskog L: Risks of solid cancers in patients with rheumatoid arthritis and after treatment with tumour necrosis factor antagonists. Ann Rheum Dis 2005, 64:1421-1426.

3. Chen YJ, Chang YT, Wang CB, Wu CY: The risk of cancer in patients with rheumatoid arthritis: a nationwide cohort study in Taiwan. Arthritis Rheum 2011, 63:352-358.

4. Mellemkjaer L, Linet MS, Gridley G, Frisch M, Moller H, Olsen JH: Rheumatoid arthritis and cancer risk. Eur J Cancer 1996, 32A:1753-1757.

5. Abasolo L, Judez E, Descalzo MA, Gonzalez-Alvaro L, Jover JA, Carmona L: Cancer in rheumatoid arthritis: occurrence, mortality, and associated factors in a South European population. Semin Arthritis Rheum 2008, 37:388-397.

6. Thomas E, Brewster DH, Black RJ, Macfarlane GJ: Risk of malignancy among patients with rheumatic conditions. Int I Cancer 2000, 88:497-502.

7. Geborek P, Bladstrom A, Turesson C, Gulfe A, Petersson IF, Saxne T, Olsson $H$, Jacobsson LT: Tumour necrosis factor blockers do not increase overall tumour risk in patients with rheumatoid arthritis, but may be associated with an increased risk of lymphomas. Ann Rheum Dis 2005, 64:699-703.

8. Baecklund E, Ekbom A, Sparen P, Feltelius N, Klareskog L: Disease activity and risk of lymphoma in patients with rheumatoid arthritis: nested case-control study. BMJ 1998, 317:180-181.

9. Arber N, Levin B: Chemoprevention of colorectal neoplasia: the potential for personalized medicine. Gastroenterology 2008, 134:1224-1237.
10. Thun MJ, Henley SJ, Patrono C: Nonsteroidal anti-inflammatory drugs as anticancer agents: mechanistic, pharmacologic, and clinical issues. J Natl Cancer Inst 2002, 94:252-266.

11. Salloum E, Cooper DL, Howe G, Lacy J, Tallini G, Crouch J, Schultz M, Murren J: Spontaneous regression of lymphoproliferative disorders in patients treated with methotrexate for rheumatoid arthritis and other rheumatic diseases. J Clin Oncol 1996, 14:1943-1949.

12. Balkwill F: TNF-alpha in promotion and progression of cancer. Cancer Metastasis Rev 2006, 25:409-416.

13. Larmonier N, Cathelin D, Larmonier C, Nicolas A, Merino D, Janikashvili $N$, Audia S, Bateman A, Thompson J, Kottke T, Hartung T, Katsanis E, Vile R, Bonnotte B: The inhibition of TNF-alpha anti-tumoral properties by blocking antibodies promotes tumor growth in a rat model. Exp Cell Res 2007, 313:2345-2355.

14. Bongartz T, Warren FC, Mines D, Matteson EL, Abrams KR, Sutton AJ: Etanercept therapy in rheumatoid arthritis and the risk of malignancies: a systematic review and individual patient data meta-analysis of randomised controlled trials. Ann Rheum Dis 2009, 68:1177-1183.

15. Bongartz T, Sutton AJ, Sweeting MJ, Buchan I, Matteson EL, Montori V: Anti-TNF antibody therapy in rheumatoid arthritis and the risk of serious infections and malignancies: systematic review and meta-analysis of rare harmful effects in randomized controlled trials. JAMA 2006, 295:2275-2285.

16. Lopez-Olivo MA, Tayar JH, Martinez-Lopez JA, Pollono EN, Cueto JP, Gonzales-Crespo MR, Gonzales-Crespo MR, Fulton S, Sauraz-Almazor ME: Risk of malignancies in patients with rheumatoid arthritis treated with biologic therapy: a meta-analysis. JAMA 2012, 308:898-908.

17. Askling J, Fahrbach K, Nordstrom B, Ross S, Schmid CH, Symmons D: Cancer risk with tumor necrosis factor alpha (TNF) inhibitors: meta-analysis of randomized controlled trials of adalimumab, etanercept, and infliximab using patient level data. Pharmacoepidemiol Drug Saf 2011, 20:119-130.

18. Wolfe F, Michaud K: Biologic treatment of rheumatoid arthritis and the risk of malignancy: analyses from a large US observational study. Arthritis Rheum 2007, 56:2886-2895.

19. Askling J, van Vollenhoven RF, Granath F, Raaschou P, Fored CM, Baecklund E, Dackhammar C, Feltelius N, Cöster L, Geborek P, Jacobsson LT, Lindblad S, Rantapää-Dahlqvist S, Saxne T, Klareskog L: Cancer risk in patients with rheumatoid arthritis treated with anti-tumor necrosis factor alpha therapies: does the risk change with the time since start of treatment? Arthritis Rheum 2009, 60:3180-3189.

20. Wu CY, Chen YJ, Ho HJ, Hsu YC, Kuo KN, Wu MS, Lin JT: Association between nucleoside analogues and risk of hepatitis $B$ virus-related hepatocellular carcinoma recurrence following liver resection. JAMA 2012, 308:1906-1914.

21. Arnett FC, Edworthy SM, Bloch DA, McShane DJ, Fries JF, Cooper NS, Healey LA, Kaplan SR, Liang MH, Luthra HS, Medsger TA Jr, Mitchell DM, Neustadt DH, Pinals RS, Schaller JG, Sharp JT, Wilder RL, Hunder GG: The American Rheumatism Association 1987 revised criteria for the classification of rheumatoid arthritis. Arthritis Rheum 1988, 31:315-324.

22. National Health Insurance Payment System, National Health Insurance Adminstration, Ministry of Health and Welfare. [http://www.nhi.gov.tw/]

23. Tsan YT, Lee CH, Wang JD, Chen PC: Statins and the risk of hepatocellular carcinoma in patients with hepatitis B virus infection. J Clin Oncol 2012, 30:623-630.

24. Chen HP, Shieh JJ, Chang CC, Chen TT, Lin JT, Wu MS, Wu CY: Metformin decreases hepatocellular carcinoma risk in a dose-dependent manner: population-based and in vitro studies. Gut 2013, 62:606-615.

25. Ruan PK, Gray RJ: Analyses of cumulative incidence functions via non-parametric multiple imputation. Stat Med 2008, 27:5709-5724.

26. Kuk $D$, Varadhan R: Model selection in competing risks regression. Stat Med 2013, 32:3077-3088.

27. cmprsk: Subdistribution Analysis of Competing Risks. [http://cran.rproject.org/web/packages/cmprsk/index.html]

28. Mercer LK, Lunt M, Low ALS, Dixon WG, Watson KD, Symmons DPM, Hyrich $\mathrm{KL}$, BSRBR Control Centre Consortium: Risk of solid cancer in patients exposed to anti-tumor necrosis factor therapy: results from the British Society for Rheumatology Biologics Register for Rheumatoid Arthritis. Ann Rheum Dis 2014, doi: 10.1136/annrheumdis-2013-204851.

29. Lan JL, Shou SJ, Chen DY, Chen YH, Hsieh TY, Young M Jr: A comparative study of etanercept plus methotrexate and methotrexate alone in Taiwanese patients with active rheumatoid arthritis: a 12-week, double- 
blind, randomized, placebo-controlled study. J Formos Med Assoc 2004 103:618-623.

30. Chen DY, Chou SJ, Hsieh TY, Chen YH, Chen HH, Hsieh CW, Lan JL: Randomized, double-blind, placebo-controlled, comparative study of human anti-TNF antibody adalimumab in combination with methotrexate and methotrexate alone in Taiwanese patients with active rheumatoid arthritis. J Formos Med Assoc 2009, 108:310-319.

31. Combe B, Codreanu C, Fiocco U, Gaubitz M, Geusens PP, Kvien TK, Pavelka K, Sambrook PN, Smolen JS, Khandker R, Singh A, Wajdula J, Fatenejad S, Etanercept European Investigators Network: Efficacy, safety and patientreported outcomes of combination etanercept and sulfasalazine versus etanercept alone in patients with rheumatoid arthritis: a double-blind randomized 2-year study. Ann Rheum Dis 2009, 68:1146-1152.

32. Emery $P$, Breedveld $F$, van der Heijde D, Ferraccioli G, Dougados M, Robertson D, Pedersen R, Koenig AS, Freundlich B, Combination of Methotrexate and Etanercept in Early Rheumatoid Arthritis Trial Group: Two-year clinical and radiographic results with combination etanerceptmethotrexate thearpy versus monotherapy in early rheumatoid arthritis: a two-year, double-blind, randomized study. Arthritis Rheum 2010, 62:674-682.

33. Keystone EC, Kavanaugh AF, Sharp JT, Tannenbaum H, Hua Y, Teoh LS Fischkoff SA, Chartash EK: Radiographic, clinical, and functional outcomes of treatment with adalimumuab (a human anti-tumor necrosis factor monoclonal antibody) in patients with active rheumatoid arthritis receiving concomitant methotrexate therapy: a randomized, placebocontrolled, 52-week trial. Arthritis Rheum 2004, 50:1400-1411.

34. Breedveld FC, Weisman MH, Kavanaugh AF, Cohen SB, Pavelka K, van Vollenhoven R, Sharp J, Perez JL, Spencer-Green GT: The PREMIER study: a multicenter, randomized, double-blind clinical trial of combination therapy with adalimumab plus methotrexate versus methotrexate alone or adalimumab alone in patients with early, aggressive rheumatoid artritis who had not had previous methotrexate treatment. Arthritis Rheum 2006, 54:26-37.

35. Bejarano V, Quinn M, Conaghan PG, Reece R, Keenan A-M, Walker D, Gough A, Green M, McGonagle D, Adebajo A, Jarrett S, Doherty S, Hordon L, Melsom R, Unnebrink K, Kupper H, Emery P, Yorkshire Early Arthritis Register Consortium: Effect of the early use of the anti-tumor necrosis factor adalimumab on the prevention of job loss in patients with early rheuatmoid arhritis. Arthritis Rheum 2008, 59:1467-1474.

36. Wolfe F, Michaud K: Lymphoma in rheumatoid arthritis: the effect of methotrexate and anti-tumor necrosis factor therapy in 18,572 patients. Arthritis Rheum 2004, 50:1740-1751.

37. Setoguchi S, Solomon DH, Weinblatt ME, Katz JN, Avorn J, Glynn RJ, Cook EF, Carney G, Schneeweiss S: Tumor necrosis factor alpha antagonist use and cancer in patients with rheumatoid arthritis. Arthritis Rheum 2006, 54:2757-2764.

38. Brown SL, Greene MH, Gershon SK, Edwards ET, Braun MM: Tumor necrosis factor antagonist therapy and lymphoma development: twenty-six cases reported to the Food and Drug Administration. Arthritis Rheum 2002, 46:3151-3158.

39. Burmester GR, Panaccione R, Gordon KB, Mcllraith MJ, Lacerda AP: Adalimumab: long-term safety in 23458 patients from global clinical trials in rheumatoid arthritis, juvenile idiopathic arthritis, ankylosing spondylitis, psoriatic arthritis, psoriasis and Crohn's disease. Ann Rheum Dis 2013, 72:517-524.

40. Theophile H, Schaeverbeke T, Miremont-Salame G, Abouelfath A, Kahn V, Haramburu F, Bégaud B: Sources of information on lymphoma associated with anti-tumour necrosis factor agents: comparison of published case reports and cases reported to the French pharmacovigilance system. Drug Saf 2011, 34:577-585.

41. Grivennikov SI, Greten FR, Karin M: Immunity, inflammation, and cancer. Cell 2010, 140:883-899.

42. Chou CT, Pei L, Chang DM, Lee CF, Schumacher HR, Liang MH: Prevalence of rheumatoid diseases in Taiwan: a population study of urban, suburban and rural differences. J Rheumatol 1994, 21:302-306.

43. Beasley RP, Bennett PH, Lin CC: Low prevalence of rheumatoid arthritis in Chinese. Prevalence survey in a rural community. J Rheumatol Supp/ 1983, 10:11-15.

44. Shickikawa K, Inoue K, Hirota S, Maeda A, Ota H, Kimura M: Changes in the incidence and prevalence of rheumatoid arthritis in Kamitonda, Kawayama, Japan, 1965-1996. Ann Rheum Dis 1999, 58:751-756.
45. Kim YJ, Shim JS, Choi CB, Bae SC: Mortality and incidence of malignancy in Korean patients with rheumatoid arthritis. J Rheumatol 2012, 39:226-232

46. Chen YJ, Chang YT, Shen JL, Chen TT, Wang CB, Chen CM, Wu CY: Association between systemic antipsoriatic drugs and cardiovascular risk in patients with psoriasis with or without psoriatic arthritis: a nationwide cohort study. Arthritis Rheum 2012, 64:1879-1887.

47. Mariette X, Matucci-Cerinic M, Pavelka K, Taylor P, van Vollenhoven R, Heatley R, Walsh C, Lawson R, Reynolds A, Emery P: Malignancies associated with tumor necrosis factor inhibitors in registries and prospective observational studies: a systemic reiew and meta-analysis. Ann Rheum Dis 2011, 70:1895-1904.

48. De Giorgi V, Gandini S, Grazzini M, Benemei S, Marchionni N, Geppetti P: Effect of $\beta$-blockers and other antihypertensive drugs on the risk of melanoma recurrence and death. Mayo Clin Proc 2013, 88:1196-1203.

49. Sørensen GV, Ganz PA, Cole SW, Pedersen LA, Sørensen HT, Cronin-Fenton DP, Garne JP, Christiansen PM, Lash TL, Ahern TP: Use of $\beta$-blockers, angiotensin-converting enzyme inhibitors, angiotensin II receptor blockers, and risk of breast cancer recurrence: a Danish nationwide prospective cohort study. J Clin Oncol 2013, 31:2265-2272.

50. Ji Y, Chen S, Xiao X, Zheng S, Li K: $\beta$-blockers: a novel class of antitumor agents. Onco Targets Ther 2012, 5:391-401.

doi:10.1186/s13075-014-0449-5

Cite this article as: Wu et al:: The risk of cancer in patients with rheumatoid arthritis taking tumor necrosis factor antagonists: a nationwide cohort study. Arthritis Research \& Therapy 2014 16:449.

\section{Submit your next manuscript to BioMed Central and take full advantage of:}

- Convenient online submission

- Thorough peer review

- No space constraints or color figure charges

- Immediate publication on acceptance

- Inclusion in PubMed, CAS, Scopus and Google Scholar

- Research which is freely available for redistribution 